\title{
Negative Pressure Wound Therapy in the Treatment of Chylousfistula after Neck Dissection
}

\author{
Binkova Hana, MD¹, Reška Michal, MD², Kostrica Rom, MD1, Horakova Zuzana, MD1 \\ ${ }^{1}$ Department of Otorhinolaryngology and Head and Neck Surgery, St. Anne's University Hospital and \\ Faculty of Medicine, Masaryk University Brno, Czech Republic \\ ${ }^{2}$ Department of General Surgery, St. Anne's University Hospital and Faculty of Medicine, Masaryk \\ University Brno, Czech Republic
}

\begin{abstract}
:
Introduction: Chylorrhoea is an uncommon but potentially dangerous complication of neck surgery, particularly neck dissection. A periperative thoracic duct suture is performed or fibrin glue, absorbable cellulose or muscle flap may be used. Postoperatively conservative therapy is preferred.

Case Report: We present a case of chylorrhoea after a salvage neck dissection persisting even after 2 surgical revisions. Chylorrhoea was successfully managed only after the use of NPWT (negative pressure wound therapy), which is so far relatively rare in otorhinolaryngological surgery.

Conclusion: The modern method of local therapy by vacuum assisted closure appears to be useful for the chylous fistula treatment as well as for secondary healing of wounds, especially after oncological salvage surgery performed in the field of post radiation fibrosis.
\end{abstract}

Keywords: chylous fistula, neck dissection, vacuum therapy, negative pressure wound therapy, NPWT

\section{INTRODUCTION}

Cervical chylorrhoea due to perforated thoracic duct (TD) is a very unpleasant complication of some neck procedures, especially neck dissection (ND). In case of massive chylous fistula (CHF) a patient loses protein, electrolytes and lipids, which significantly prolongs the healing process. The wound is often complicated by inflammation, necrosis and major neck vessels are at risk of rupture. The treatment consensus is not clear.

We present a case of massive chylorrhoea after the salvage ND, where repeated surgical explorations had failed and finally NPWT was successfully applied.

\section{CASE REPORT}

A 55-year-old man with primary cutaneous adnexal carcinoma of right nuchal area with cervical metastases was treated by radical ND followed by radiotherapy (RT). After 6 months salvage ND was performed on the left due to metastatic supraclavicular lymphnodes. The procedure was without intraoperative complications, chylorrhoea unseen. For post-RT laryngeal swelling, the patient received a prophylactic tracheostomy. On the second postoperative day (PD) there was a visible milky turbid secretion in the drains, with waste increasing up to $1000 \mathrm{ml}$ in subsequent days. No effect of supraclavicular compression and fat free diet was observed. On the 9th PD the first exploration with the TD ligation was performed. After 3 days the chylorrhoea $(500-1300 \mathrm{ml} /$ day) reappeared. As total parenteral nutrition was without effect, on the 18th PD a second surgical revision was performed. Leaking lymphatic vessels were identified under microscopic control (without preoperative lymphangiography) and resutured. The exposed field was overlapped with absorbable cellulose mesh (Surgicel) and fibrin glue (Tissucol). Unfortunately, this operation was also unsuccessful. Skin necrosis ranged from $3 \times 4 \mathrm{~cm}$, the chylus loss was 500 to $800 \mathrm{ml} /$ day [Fig. 1]. There was only mild hypoalbuminemia $2,7 \mathrm{~g} / \mathrm{dl}$ in laboratory finding. In cooperation with the surgical department, vacuum therapy was implemented using an NPWT device for further healing. Polyurethane foam and suction drain were inserted and sealed with foil and negative continuous pressure of -85 to $-100 \mathrm{mmHg}$ was 
applied [Fig. 2]. NPWT was well tolerated. From the beginning the drain waste was at a minimum, at the first dressing changes after 3 days the chyle was not leaking, the wound was granulating and the defect size within 20 days (6x changed set) of NPTW application was reduced to less than half. The defect epithelialization was completed without NPWT [Fig. 3].

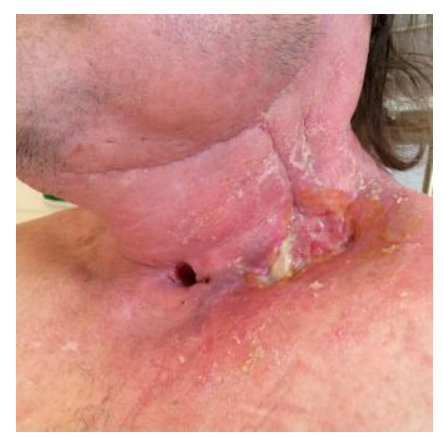

Fig1. Supraclavicular skin defect with chylous leakage

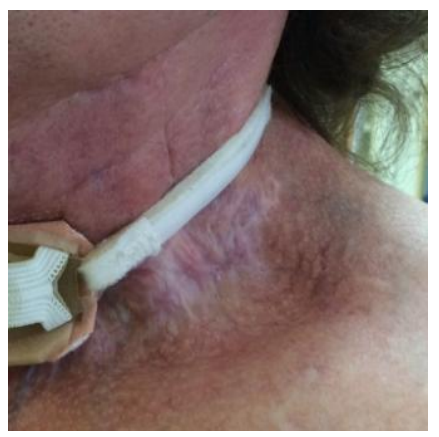

Fig.2: Skin defect with chylous fistula closed by polyurethane foam, transparent coverage and the drain ensuring drainage and vacuum by connection with vacuum pump

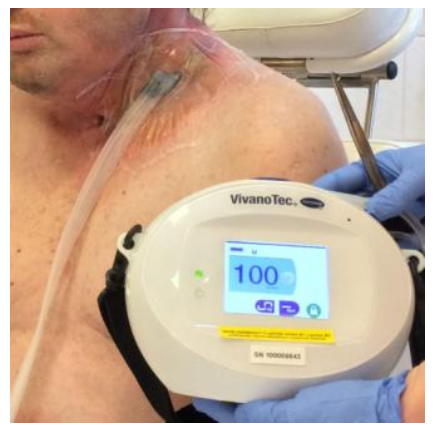

Fig3. Healedwound 6 weeksafter NPWT

\section{DisCUSSION}

$\mathrm{CHF}$ is an uncommon but potentially dangerous complication of a neck surgery. The most risky procedure is ND, in particular radical ones which is accompanied by chylorrhoea in $1-2 \%$, in $75 \%$ on the left[1, 2]. Also risky is total thyroidectomy[3], subclavian vein cannulation or injury. Incase of intraoperative leakage, a surgeon is supposed to identify the place of injury and close the perforation. Where a fragile duct suture is impossible, cellulose mesh, fibrin glue or muscle flaps (sternohyoid or sternocleidomastoideus) may be used in the fistula closure. For postoperative chyle leaks a conservative therapy is preferred [4,5] : bed rest, drainage, compression bandages, medium chain triglycerides diet, total parenteral nutrition. Low-output $(<500 \mathrm{ml} / 24 \mathrm{~h})$ fistulas tend to respond to conservative measures, whereas high-output secreting fistulas $(>500 \mathrm{ml} / 24 \mathrm{~h})$ are generally refractory and require surgical revision.

NPWT has been used in the treatment of open wounds of various etiologies since 1995. A portable pump creates negative pressure which is transmitted via a drainage system to the wound base. The moist wound healing in a closed environment, progressive reduction in the of wound volume by drawing its edge, continuous drainage of exudate and tissue fluid and promotion of granulation provide the greatest advantages. The vacuum may be applied continuously or intermittently, the vacuum level (usually between -125 and $-75 \mathrm{mmHg}$ ) depends on the device, material and the patient's 
tolerance $[6,7]$. Coverage is usually changed twice or three times a week. Some NPWT devices allow delivery of fluids into the wound which can be moistened with saline or antibiotics. Given its very good clinical outcomes, it has become the gold standard treatment of wound dehiscence (especially sternotomy and laparotomy).

This method has been completely neglected in otorhinolaryngology until recently. Publications (rather case reports) confirm the positive effects of the therapy, but high-quality, reliable studies are still missing [8]. Experience with a set of 115 patients (2005 to 2011) was published by Asher [9]. In almost $80 \%$, wounds were risky and complicated (saliva contamination, exposure bones, exposed major vessels, the previous free flap transfer, peritracheostomy application after total laryngectomy), and two-thirds of patients had pre-existing risk factors (previous radiotherapy, diabetes mellitus, hypothyroidism). Adverse effects occurred in only $3.5 \%$ of the patients. The method was evaluated as safe and potentially very useful for the complicated wound healing in HN. Even other American authors recommend NPWT be included into routine otolaryngology surgical methods for complicated wound treatment. 84\% of patients with NPWT (total 19) avoided secondary surgery [10].

NPWT in the chylous fistula treatment was described only once, as in our case in the patient after ND [11]. The low output chylorrhoea appeared on the fourth postoperative day and NPWT was initiated on the ninth day afterward. The fistula closed within 6 days. In our case conservative treatment demanding two surgical revisions with sutures of the duct, using fibrin glue, cellulose mesh and muscle flap was not effective. The pump was applied on the 33th PD, in three days massive secretion ceased, within 20 days the wound was significantly reduced. One of the NPWT limits is the exposure of large blood vessels and nerves, where the risk of vein rupture must be considered [7]. In our case, the large vessels, after previous surgical intervention, were covered by absorbable cellulose and muscle and granulation tissue. NPWT seems to be a very useful method in cervical chylous fistula healing. However, due to jugular vein and carotid artery proximity, NPWT must be used with caution. According to our experience it is convenient to include vacuum treatment in the management of the treatment of chylous fistula before it becomes the last option after using up all other methods. The patient could be spared non effective surgical procedures and with shorter healing time.

In conclusion, nowadays vacuum therapy has been proved to be an effective and reliable method of wound healing in surgery. However its use in otorhinolaryngology is still very sporadic and in the treatment of chylous fistula after injury of TD, absolutely rare. Our case report shows that NPWT seems to be not only a useful tool for the chylous fistula treatment, but also for other cases of complicated wounds in $\mathrm{HN}$, especially in the secondary healing treatment after salvage oncosurgery in the field of post radiation fibrosis.

\section{ACKNOWLEDGMENTS}

Theworkwassupported by followingprojects:the Ministry ofHealthof CR (1629835A), and the Czech Science Foundation (16-12454S).

\section{REFERENCES}

[1] Crumley RL, Smith JD. Postoperative chylous fistula- prevention and management. Laryngoscope. 1976; 86: 804-13

[2] Campisi CC, Boccardo F, Piazza C, Campisi C. Evolution of chylous fistula management after neck dissection. CurrOpinOtolaryngol Head Neck Surg. 2013 Apr;21(2):150-6

[3] Shivakumar T, Ashok MS, Prashanth V et al. Massive chylorrhea following total thyroidectomy and neck dissection. International Journal of Head and Neck Surgery. 2012; 3(1):45

[4] Chung E-J, Baek S-K, Kwon S-Y, Woo J-S, Jung K-Y. Moderately Differentiated Neuroendocrine Carcinoma of the Larynx. Clinical and Experimental Otorhinolaryngology. 2008;1(4):217-220

[5] Spiro JD., Spiro RH, Strong EW. The management of chyle fistula. Laryngoscope. 1990; 100:771-74

[6] Morykwas MJ, Argenta LC, Shelton-Brown ET et al. Vacuum-assisted closure: a new method for wound control and treatment. Annals of Plastic Surgery.1997; 38 (6): 553-62

[7] Orgill DP, Manders EK, Sumpio BE et al. The mechanisms of action of vacuum assisted closure: more to learn. Surgery. 2009 Jul; 146(1):40-51 
Binkova Hana et al.

[8] Palm HG, Hauer T, Simon C, Willy C. Vacuum-assisted closure of head and neck wound. HNO. 2011 Aug;59(8):819-30

[9] Asher SA, White HN, Golden JB et al. Negative pressure wound therapy in head and neck surgery. JAMA Facial Plast Surg. 2014; 16(2):120-6

[10] Dhir K, Reino AJ, Lipana J. Vacuum-assisted closure therapy in the management of head and neck wounds.Laryngoscope.2009; 119:(1) 54-61

[11] Kadota H, Kakiuch IY, Yoshida T. Management of chylous fistula after neck dissection using negative-pressure wound therapy: A preliminary report. Laryngoscope. 2012; 122(5):997-9 